\title{
Risk factors and population attributable risk percent for lactation mastitis in Chinese women: a systematic review and meta-analysis
}

Baoyong Lai ( $\sim$ by_lai@126.com )

Beijing University of Chinese Medicine https://orcid.org/0000-0002-2874-4066

\section{Bo-Wen Yu}

Beijing University of Chinese Medicine

\section{Ai-Jing Chu}

Beijing University of Chinese Medicine

\section{Shi-Bing Liang}

Beijing University of Chinese Medicine

Li-Yan Jia

Beijing University of Chinese Medicine

Jian-Ping Liu

Beijing University of Chinese Medicine

\section{Ying-Yi Fan \\ Beijing University of Chinese Medicine}

Xiao-Hua Pei

Beijing University of Chinese Medicine

\section{Research article}

Keywords: Lactation mastitis, Risk factors, Population attributable risk percent, Meta-analysis

Posted Date: September 30th, 2020

DOI: https://doi.org/10.21203/rs.3.rs-83204/v1

License: @ (i) This work is licensed under a Creative Commons Attribution 4.0 International License. Read Full License 


\section{Abstract}

Background: Lactation mastitis (LM) is one of the most common breast disorders experienced by postpartum women, affecting approximately $3 \%$ to $20 \%$ breastfeeding mothers. Although a few researches have studied the risk factors related to LM, there are still some inconsistent problems on this topic and nationally representative evidence is scarce. This study was aimed to determine the wellaccepted risk factors for LM in Chinese women.

Methods: Six electronic literature databases were searched from their inception to June 1st, 2020. We used RevMan5.3 and Stata14.0 for data analysis. The strength of the association was summarized using odds ratio (OR) with $95 \%$ confidence intervals (Cl). The population attributable risk (PAR) percent was calculated for significant risk factors. The fail-safe number (Nfs) was used to estimate publication bias and robustness of the current results.

Results: Twelve studies involving 6539 participants were included. A total of 18 potential risk factors were eventually evaluated. Significant risk factors for LM included improper breastfeeding posture (OR 2.47, 95\% Cl 2.09-2.92; PAR 26.51\%), improper milking method (OR 6.79, 95\% Cl 3.45-13.34; PAR 59.14\%), repeated milk stasis (OR 6.08, 95\% Cl 3.75-9.88; PAR 37.42\%), little or no cleaning of nipples (OR 2.38, 95\% Cl 1.88-3.03; PAR 31.28\%), abnormal nipple or crater nipple (OR 2.94, 95\%Cl 1.76-4.91; PAR 33.99\%), primipara (OR 1.91, 95\% Cl 1.26-2.90; PAR 39.06\%), history of breast trauma (OR 3.57, 95\% Cl 2.86-4.46; PAR 13.36\%), experience of cesarean section (OR 1.77, 95\% Cl 1.32-2.38; PAR 28.34\%), low educational level (OR 1.85, 95\% Cl 1.04-3.28, PAR 23.67\%), breast massage experience of non-medical personnel (OR 1.90, 95\% Cl 1.36-2.65, PAR 20.89\%), postpartum within 6 months (OR 5.11, 95\% Cl 2.66-9.82; PAR 65.93\%), prone sleeping position (OR 2.53,95\% Cl1.51-4.22; PAR 22.72\%) and postpartum rest time less than 3 months (OR 4.71, $95 \% \mathrm{Cl} 3.92-5.65$; PAR $56.95 \%$ ). There seemed to be good robustness of the pooled results for most of the included risk factors.

Conclusion: This study found several important risk factors of LM in Chinese women. In particular, the incidence of LM can be reduced by controlling several modifiable factors such as breastfeeding posture, milking method, milk stasis situation and nipple cleaning condition, etc.

\section{Background}

Lactation mastitis (LM) is one of the most common diseases in postpartum women [1]. It is clinically characterized by a red, swollen, hot and tender area of breast, probably accompanied by high fever, headache, and other influenza-like symptoms [2]. The incidence of LM varied widely across populations and up to approximately $3-20 \%$ of breastfeeding mothers may suffer from LM in their lifetime [3, 4]. LM occurs frequently in the first six to eight weeks of postpartum but it can also occur at any time during breastfeeding. Besides, it was reported that about one-third of women could experience a recurrent episode [5]. In addition, previous studies have shown that mismanagement or incorrect breast care can lead to the development of LM into severe cases (such as breast abscess or sepsis), which would directly lead to the cessation of normal breastfeeding $[3,6]$.

The World Health Organization (WHO) or international guidelines highly recommended breastfeeding for all infants within six months after birth as a natural way of infant feeding, because it can provide the best nutritional starting point for infants and promote their healthy growth $[1,7,8]$. Additionally, breastfeeding has been reported to have beneficial effects on the health outcomes of both infants and mothers. However, it is of concern that previous surveys in Australia reported less than $15 \%$ of women exclusively breastfeed their five-month-old infants [9]. In china, similar results show that the breastfeeding rate of infants aged 1-2 months ranges from 59.4$66.5 \%[10,11]$. In addition, it was reported that the main causes directly inducing breastfeeding failure were LM and its related discomfort [12]. Therefore, it is of great significance to explore the risk factors associated with LM and prolong lactation.

Although a few researches have studied the risk factors related to $L M$, there are still some inconsistent problems in LM risk factors due to the complexity of LM etiology. These problems have an important impact on the management of breast care $[13,14,15]$. Therefore, it is critical and necessary for lactating mothers to detect and avoid the high risk factors associated with LM. However, there is no research systematically summarizing the significant risk factors associated with LM among Asian women. In addition, extensive search of Chinese and English literature has not found any quantitative meta-analysis to assess the risk factors associated with LM in Chinese women. To provide nationally representative evidence to the well-accepted risk factors for LM, we performed this systematic review to determine the corresponding significant risk factors related to LM. Furthermore, to estimate the potential impact of these factors on LM at the population level, the population attributable risk (PAR) percent, was calculated where possible. Doctors and lactating mothers can identify the high risk factors of $L M$, which is helpful to reduce the incidence of LM. 


\section{Methods}

A systematic review and meta-analysis of relevant studies was conducted and reported, following the PRISMA recommendations. The protocol of this review has been registered at PROSPERO (CRD42020186674).

\subsection{Eligibility/exclusion Criteria}

The following criteria were used to identify relevant studies: (1) This review included case-control studies, cohort studies, crosssectional studies and randomized controlled trials (RCTs) to explore the risk factors associated with LM; (2) All considered participants were Chinese women, regardless of their age or race; and (3) English and Chinese language publications.

Studies were excluded from the analysis: (1) data could not be extracted; (2) Studies where the outcome was not clearly stated and (3) Studies that included duplicate data.

\subsection{Search Strategy}

We systematically searched PubMed, Web of science, Chinese Biomedical Literature Database (SinoMed), China National Knowledge Infrastructure (CNKI), Wan fang Database and China Science Technology Journal Database (VIP) from their inception to June 1st, 2020. The following search terms were used, including lactation mastitis, acute mastitis, risk factors, influence factors and factor analysis.

\subsection{Study Selection And Data Extraction}

Two authors independently selected the studies and extracted the detailed data of the eligible trials. The items for data extraction were first authors, year of publication, study type, the detailed information of methodology, characteristics of participants, sample size, the data of risk factors associated with LM, and the incidence of LM, etc. Any discrepancies regarding study selection and data extraction was resolved through consensus and arbitrated by the third author if necessary.

\subsection{Quality Assessment}

The quality of case-control studies and cohort studies were assessed according to the criteria of Newcastle-Ottawa Scale (NOS) [16]. The "star" scoring system of NOS was used during evaluation process and a star was described as an appropriate entry, with each star representing one point. The possible NOS assessment score ranged from zero to nine points. The Agency for Healthcare Research and Quality (ARHQ) methodology checklist was used to evaluate the quality of the studies that were cross-sectional study [17]. We evaluated the quality of RCTs by using Cochrane risk of bias tool. Any disagreements were resolved by discussion with a third author.

\subsection{Statistical Analysis}

We used RevMan5.3 and Stata14.0 to perform statistical analysis, binary data were summarized using odds ratio (OR) with their $95 \%$ confidence intervals $(\mathrm{Cl})$. We assessed statistical heterogeneity by using the $R^{2}$ statistics test and Q chi-squared test. When $R^{2}$ result > $50 \%$ and $Q$ chi-squared test result $<0.10$, it shows that there is significant statistical heterogeneity among the trials $\llbracket$ and the random effects model was adopted. Otherwise, it shows that there is no obvious statistical heterogeneity among the trials, and the fixed effects model was used. Sensitivity analysis was performed when possible to test the robustness of the results.

The PAR percent were calculated to indicate the proportion of cases that can be attributed to each risk factor according to the following formula [18].

$$
P A R=\frac{P_{e}(O R-1)}{1+P_{e}(O R-1)}
$$

The PAR percent is calculated using the pooled OR for each risk factor, and is estimated based on the identified meta-analysis. ' $P e^{\prime}$ is the prevalence of exposure in the population.

The fail-safe number (Nfs) was calculated to measure publication bias according to the following formula. $\mathrm{Nfs} 0.05=\left(\sum \mathrm{Z} / 1.64\right)^{2}-\mathrm{K}$, $\mathrm{Nfs} 0.01=\left(\sum \mathrm{Z} / 2.33\right)^{2}-\mathrm{K}$, the $\mathrm{K}$ in formula is the number of selected studies. The larger the value of $\mathrm{Nfs}$, the smaller the bias [19]. 
Additionally, Nfs value was used to estimate the strength of the current evidence by calculating the number of negative studies required to nullify our conclusions. Furthermore, Egger's linear regression tests were performed to further evaluate publication bias.

\section{Results}

\subsection{The selection of study}

A total of 215 related articles were obtained from 6 databases. First duplicates were excluded, and then 136 articles were excluded by reading the title and abstract. Full texts of 26 articles were screened according to the eligibility criteria. Finally, twelve articles (12 trials) met inclusion criteria that were included for analysis. The selection process was showed in Fig. 1.

\subsection{Study Characteristics And Quality Assessment Of Included Studies}

In total, twelve studies were included, involving a combined total of 6539 participants. One of the studies [20] was a prospective cohort study, two of which $[30,31]$ were cross-sectional studies and the rest were case-control studies. Ten of the included studies were

published in Chinese [22-31], and two studies were published in English [20,21]. The detailed characteristic of the included studies was shown in Table 1. 
Table 1

Basic information of included studies

\begin{tabular}{|c|c|c|c|c|c|c|c|c|}
\hline \multirow[t]{2}{*}{ Study ID } & \multirow{2}{*}{$\begin{array}{l}\text { Research } \\
\text { design } \\
\text { type }\end{array}$} & \multirow[t]{2}{*}{ Area/Provence } & \multicolumn{2}{|c|}{$\begin{array}{l}\text { Sample size } \\
\text { (n) }\end{array}$} & \multicolumn{2}{|c|}{ age(years) } & \multirow[t]{2}{*}{ Risk factors } & \multirow{2}{*}{$\begin{array}{l}\text { NOS/ARHQ } \\
\text { (score) }\end{array}$} \\
\hline & & & $\mathbf{T}$ & C & $\mathrm{T}$ & C & & \\
\hline $\begin{array}{l}\mathrm{Li} \\
\text { T2014[20] }\end{array}$ & $\begin{array}{l}\text { Cohort } \\
\text { study }\end{array}$ & Sichuan & 42 & 628 & 24 & 24 & $4,813,15,16,18$ & 8 \\
\hline $\begin{array}{l}\text { Zhong HY } \\
\text { 2018[21] }\end{array}$ & $\begin{array}{l}\text { Case } \\
\text { control } \\
\text { study }\end{array}$ & Shandong & 63 & 262 & NR & NR & $10,11,15$ & 6 \\
\hline $\begin{array}{l}\mathrm{He} \\
\text { XP2013[22] }\end{array}$ & $\begin{array}{l}\text { Case } \\
\text { control } \\
\text { study }\end{array}$ & Beijing & 237 & 237 & $\begin{array}{l}29.9 \\
\pm 3.0\end{array}$ & $\begin{array}{l}27.3 \\
\pm 3.6\end{array}$ & $4,5,6,8,9,13,14$ & 8 \\
\hline $\begin{array}{l}\text { Pu } \\
\text { YN2017[23] }\end{array}$ & $\begin{array}{l}\text { Case } \\
\text { control } \\
\text { study }\end{array}$ & Zhejiang & 1000 & 1000 & NR & NR & $1,2,3,4,5,6,7,8,9,14,18$ & 7 \\
\hline $\begin{array}{l}\text { Li } \\
\text { JX2019[24] }\end{array}$ & $\begin{array}{l}\text { Case } \\
\text { control } \\
\text { study }\end{array}$ & Guangdong & 135 & 135 & NR & NR & $4,5,6,8,9,10,12,13,15,16,17$ & 7 \\
\hline $\begin{array}{l}\text { Wang } \\
\text { HM2016[25] }\end{array}$ & $\begin{array}{l}\text { Case } \\
\text { control } \\
\text { study }\end{array}$ & Fujian & 241 & 241 & $\begin{array}{l}27.5 \\
\pm 5.63\end{array}$ & $\begin{array}{l}31.2 \\
\pm 5.0\end{array}$ & $1,4,6,10,11,13,14,17,18$ & 8 \\
\hline $\begin{array}{l}\text { Cheng } \\
\text { MH2014[26] }\end{array}$ & $\begin{array}{l}\text { Case } \\
\text { control } \\
\text { study }\end{array}$ & Guangdong & 100 & 100 & NR & NR & $1,2,3,5,7,8,15,18$ & 7 \\
\hline $\begin{array}{l}\text { Zhai } \\
\text { HL2017[27] }\end{array}$ & $\begin{array}{l}\text { Case } \\
\text { control } \\
\text { study }\end{array}$ & Henan & 224 & 224 & $\begin{array}{l}28.61 \\
\pm 3.05\end{array}$ & $\begin{array}{l}29.24 \\
\pm 3.19\end{array}$ & $4,5,6,7,8,12,13,14$ & 7 \\
\hline $\begin{array}{l}\text { Gao } \\
\text { X2015[28] }\end{array}$ & $\begin{array}{l}\text { Case } \\
\text { control } \\
\text { study }\end{array}$ & Chongqing & 100 & 100 & $\begin{array}{l}29.33 \\
\pm 9.20\end{array}$ & $\begin{array}{l}29.12 \\
\pm 8.35\end{array}$ & $4,5,6,8,9,13,14$ & 7 \\
\hline $\begin{array}{l}\text { Chen } \\
\text { XG2016[29] }\end{array}$ & $\begin{array}{l}\text { Case } \\
\text { control } \\
\text { study }\end{array}$ & Guangdong & 313 & 267 & NR & NR & $1,5,6,11,15,16$ & 6 \\
\hline $\begin{array}{l}\text { Xia } \\
\text { HL2011[30] }\end{array}$ & $\begin{array}{l}\text { Cross } \\
\text { sectional } \\
\text { study }\end{array}$ & Jiangsu & 846 & & NR & & 15,18 & 9 \\
\hline $\begin{array}{l}\text { Wang } \\
\text { XL2018[31] }\end{array}$ & $\begin{array}{l}\text { Cross } \\
\text { sectional } \\
\text { study }\end{array}$ & Shanxi & 68 & & 29.14 & .36 & 9 & 6 \\
\hline \multicolumn{9}{|c|}{$\begin{array}{l}\text { Note: T: Case group, C: Control group, NR: Not reported, NOS: Newcastle-Ottawa Scale, ARHQ: The Agency for Healthcare Research } \\
\text { and Quality. Risk factors: } 1 \text { - history of breast disease, 2-milking method, 3- rest time of postpartum women, 4-nipple cleaning } \\
\text { condition, } 5 \text { - the way baby sucks nipple, 6- history of breast trauma, 7-breastfeeding posture, 8-postpartum bad mood, 9- sleeping } \\
\text { posture after delivery, } 10 \text {-history of diabetes, } 11 \text {-abnormal nipple or crater nipple, 12-breast massage experience of non-medical } \\
\text { personnel, } 13 \text { - the duration of each breastfeeding, 14- postpartum period, 15- primipara. 16-method of delivery, 17- repeated milk } \\
\text { stasis, 18-education level. }\end{array}$} \\
\hline
\end{tabular}

According to the NOS of the case-control study, the quality of all the included studies was evaluated from three aspects: the selection of subjects, comparability and exposure. The total scores of all case-control studies were from medium to high quality, mainly due to comparability and exposure (with insufficient definition of control and non-response rate). According to the NOS of cohort study, we evaluated the quality of one cohort study based on the object selection, comparability and outcome. The quality of the cohort study was medium, mainly due to non-response rate. According to the ARHQ on cross sectional study, we evaluated the quality of two cross sectional studies based on selection bias, performance bias, detection bias, attrition bias and reporting bias. The quality of all cross- 
sectional studies was medium or low, mainly due to detection bias and reporting bias. The overall quality of the included studies was acceptable. The score of NOS or ARHQ was shown in Table 1.

\subsection{Results Of Metaanalysis}

More than two studies involving the same LM risk factor were included in the meta-analysis. The PAR of risk factors (OR $\nabla$ ) significantly associated with LM was calculated. A total of 18 potential risk factors were identified, of which 6 were classified as breastfeeding related risk factors (including breastfeeding posture, milking method, the duration of each breastfeeding, the way baby sucks nipple, repeated milk stasis and nipple cleaning condition). Eight of them were classified as maternal related risk factors (including abnormal nipple or crater nipple, primipara, history of breast trauma, history of breast disease, history of diabetes, method of delivery, education level and breast massage experience of non-medical personnel). Four risk factors were categorized as other risk factors related to postpartum period (including postpartum period, sleeping posture after delivery, rest time of postpartum women and postpartum bad mood). The results of analyses were shown in Table 2 and Table 3. 
Table 2

effect estimates of meta-analysis

\begin{tabular}{|c|c|c|c|c|c|c|c|c|}
\hline \multirow[t]{2}{*}{ Risk factors } & \multirow{2}{*}{$\begin{array}{l}\text { Study } \\
\text { (NO.) }\end{array}$} & \multirow[t]{2}{*}{ Participant } & \multicolumn{3}{|c|}{ Heterogeneity analysis } & \multicolumn{2}{|c|}{$\begin{array}{l}\text { results of meta- } \\
\text { analysis }\end{array}$} & \multirow[t]{2}{*}{$\begin{array}{l}\text { Study } \\
\text { ID }\end{array}$} \\
\hline & & & $R$ & $\begin{array}{l}\mathrm{p}- \\
\text { value }\end{array}$ & model & $\begin{array}{l}\text { OR and } \\
\text { their } \\
95 \% \mathrm{Cl}\end{array}$ & $\begin{array}{l}\mathrm{p}- \\
\text { value }\end{array}$ & \\
\hline \multicolumn{9}{|l|}{ 1Risk factors related to breastfeeding } \\
\hline Improper breastfeeding posture & 3 & 2068 & $0 \%$ & 0.79 & FIX & $\begin{array}{l}\text { OR2.47 } \\
{[2.09} \\
2.92]\end{array}$ & $<0.001$ & $\begin{array}{l}{[23,26,} \\
27]\end{array}$ \\
\hline Improper milking method & 2 & 2160 & $69 \%$ & 0.007 & REM & $\begin{array}{l}\text { OR6.79 } \\
{[3.45,} \\
13.34]\end{array}$ & $<001$ & {$[23,26]$, } \\
\hline $\begin{array}{l}\text { The duration of each } \\
\text { breastfeeding } ₫ 0.5 \mathrm{~h}\end{array}$ & 6 & 2544 & $83 \%$ & $<001$ & REM & $\begin{array}{l}\text { OR0.77 } \\
\text { [0.48, } \\
1.24]\end{array}$ & 0.29 & $\begin{array}{l}{[20,22,} \\
24,25 \\
27,28]\end{array}$ \\
\hline Only baby sucking the nipple & 7 & 4132 & $98 \%$ & $<001$ & REM & $\begin{array}{l}\text { OR0.76 } \\
{[0.19,} \\
2.97]^{\prime}\end{array}$ & 0.69 & $\begin{array}{l}{[22-24,} \\
26-29]\end{array}$ \\
\hline Repeated milk stasis & 2 & 752 & $36 \%$ & 0.21 & FIX & $\begin{array}{l}\text { OR6.08 } \\
{[3.75,} \\
9.88]\end{array}$ & $<001$ & {$[24,25]$} \\
\hline Little or no cleaning nipple & 7 & 4544 & $52 \%$ & 0.05 & REM & $\begin{array}{l}\text { OR2.38 } \\
{[1.88} \\
3.03]\end{array}$ & $<0.001$ & $\begin{array}{l}{[20,} \\
22-25, \\
27,28]\end{array}$ \\
\hline \multicolumn{9}{|l|}{ 2Risk factors related to maternity } \\
\hline Abnormal nipple or crater nipple & 4 & 1697 & $76 \%$ & 0.06 & REM & $\begin{array}{l}\text { OR2.94 } \\
{[1.76,} \\
4.91]\end{array}$ & $<001$ & $\begin{array}{l}{[20,21} \\
25,29]\end{array}$ \\
\hline Primipara & 5 & 2005 & $60 \%$ & 0.04 & REM & $\begin{array}{l}\text { OR1.91 } \\
\text { [1.26, } \\
2.90]\end{array}$ & 0.02 & $\begin{array}{l}{[20,21,} \\
24,26, \\
29]\end{array}$ \\
\hline History of breast trauma & 6 & 3874 & $29 \%$ & 0.22 & FIX & $\begin{array}{l}\text { OR3.57 } \\
{[2.86,} \\
4.46]\end{array}$ & $<0.001$ & $\begin{array}{l}{[22-25,} \\
27,28]\end{array}$ \\
\hline History of breast disease & 4 & 2642 & $93 \%$ & $\begin{array}{l}< \\
0.01\end{array}$ & REM & $\begin{array}{l}\text { OR0.99 } \\
{[0.41,2.42]}\end{array}$ & $<001$ & $\begin{array}{l}{[23,25,} \\
26,29]\end{array}$ \\
\hline History of diabetes & 3 & 3222 & $46 \%$ & 0.16 & FIX & $\begin{array}{l}\text { OR2.26 } \\
{[1.43,3.58]}\end{array}$ & $<.001$ & $\begin{array}{l}{[21,24,} \\
25]\end{array}$ \\
\hline Experience of cesarean section & 3 & 1520 & $38 \%$ & 0.20 & FIX & $\begin{array}{l}\text { OR1.77 } \\
{[1.32,2.38]}\end{array}$ & $<001$ & $\begin{array}{l}{[20,24,} \\
29]\end{array}$ \\
\hline Low education level & 4 & 3312 & $82 \%$ & $\begin{array}{l}< \\
0.01\end{array}$ & REM & $\begin{array}{l}\text { OR1.85 } \\
{[1.04,2.38]}\end{array}$ & $<0.001$ & $\begin{array}{l}{[20,23,} \\
25,26]\end{array}$ \\
\hline $\begin{array}{l}\text { Breast massage experience of non- } \\
\text { medical personnel }\end{array}$ & 2 & 718 & $24 \%$ & 0.25 & FIX & $\begin{array}{l}\text { OR1.90 } \\
{[1.36} \\
2.65]\end{array}$ & $<001$ & {$[24,27]$} \\
\hline \multicolumn{9}{|l|}{$\begin{array}{l}\text { 30ther risk factors related to } \\
\text { postpartum period }\end{array}$} \\
\hline Postpartum within 6 months & 5 & 3604 & $93 \%$ & $\dot{0} 0.01$ & REM & $\begin{array}{l}\text { OR5.11 } \\
{[2.66,9.82]}\end{array}$ & $<0.001$ & $\begin{array}{l}{[22,23,} \\
25,27, \\
28]\end{array}$ \\
\hline
\end{tabular}

REM, random effect model; FIX, fixed effect model; OR, odds ratio; Cl, confidence interval. 


\begin{tabular}{|c|c|c|c|c|c|c|c|c|}
\hline \multirow[t]{2}{*}{ Risk factors } & \multirow{2}{*}{$\begin{array}{l}\text { Study } \\
\text { (NO.) }\end{array}$} & \multirow[t]{2}{*}{ Participant } & \multicolumn{3}{|c|}{ Heterogeneity analysis } & \multicolumn{2}{|c|}{$\begin{array}{l}\text { results of meta- } \\
\text { analysis }\end{array}$} & \multirow[t]{2}{*}{$\begin{array}{l}\text { Study } \\
\text { ID }\end{array}$} \\
\hline & & & $R$ & $\begin{array}{l}\mathrm{p}- \\
\text { value }\end{array}$ & model & $\begin{array}{l}\text { OR and } \\
\text { their } \\
95 \% \mathrm{Cl}\end{array}$ & $\begin{array}{l}\mathrm{p}- \\
\text { value }\end{array}$ & \\
\hline $\begin{array}{l}\text { The prone sleeping position after } \\
\text { delivery }\end{array}$ & 4 & 2944 & $84 \%$ & $<.001$ & REM & $\begin{array}{l}\text { OR2.53 } \\
{[1.51,4.22]}\end{array}$ & $<.001$ & $\begin{array}{l}{[22-24,} \\
28]\end{array}$ \\
\hline $\begin{array}{l}\text { Postpartum rest time less than } 3 \\
\text { months }\end{array}$ & 2 & 2160 & $0 \%$ & 0.81 & FIX & $\begin{array}{l}\text { OR4.71 } \\
{[3.92,} \\
5.65]\end{array}$ & $<001$ & {$[23,26]$} \\
\hline Postpartum bad mood & 7 & 4222 & $77 \%$ & $<.001$ & REM & $\begin{array}{l}\text { OR1.28 } \\
{[0.92,} \\
1.79]\end{array}$ & 0.15 & $\begin{array}{l}{[20,} \\
22-24, \\
26-28]\end{array}$ \\
\hline
\end{tabular}

Table 3

the results of the PAR and the Nfs

\begin{tabular}{|c|c|c|c|c|c|c|}
\hline \multirow[t]{2}{*}{ Risk factor } & \multirow[t]{2}{*}{ Study } & \multicolumn{3}{|c|}{$\begin{array}{l}\text { The population attributable risk } \\
\text { percent }\end{array}$} & \multicolumn{2}{|c|}{$\begin{array}{l}\text { The fail-safe } \\
\text { number }\end{array}$} \\
\hline & & OR & $\mathrm{Pm}(\%)$ & PAR (\%) & $\mathrm{Nfs} 0.05$ & Nfs0.01 \\
\hline \multicolumn{7}{|l|}{ 1Risk factors associated with breastfeeding } \\
\hline Improper breastfeeding posture & 3 & 2.47 & 24.54 & 26.51 & 32 & 13 \\
\hline Improper milking method & 2 & 6.79 & 25.00 & 59.14 & NA & NA \\
\hline Repeated milk stasis & 2 & 6.08 & 11.71 & 37.42 & NA & NA \\
\hline Little or no cleaning nipple & 7 & 2.38 & 32.98 & 31.28 & 126 & 59 \\
\hline \multicolumn{7}{|l|}{ 2Risk factors related to maternity } \\
\hline Abnormal nipple or crater nipple & 4 & 2.94 & 26.53 & 33.99 & 40 & 18 \\
\hline Primipara & 5 & 1.91 & 70.04 & 39.06 & 72 & 33 \\
\hline History of breast trauma & 6 & 3.57 & 5.98 & 13.36 & 92 & 43 \\
\hline History of diabetes & 3 & 2.26 & 5.79 & 6.81 & 16 & 6 \\
\hline Experience of cesarean section & 3 & 1.77 & 51.35 & 28.34 & 14 & 6 \\
\hline Low education level & 4 & 1.85 & 36.48 & 23.67 & 22 & 9 \\
\hline $\begin{array}{l}\text { Breast massage experience of non-medical } \\
\text { personnel }\end{array}$ & 2 & 1.90 & 29.25 & 20.89 & NA & NA \\
\hline \multicolumn{7}{|l|}{3 Other risk factors related to postpartum period } \\
\hline Postpartum within 6 months & 5 & 5.11 & 46.84 & 65.93 & 72 & 33 \\
\hline The prone sleeping position after delivery & 4 & 2.53 & 19.15 & 22.72 & 33 & 14 \\
\hline Postpartum rest time less than 3 months & 2 & 4.71 & 35.46 & 56.95 & NA & NA \\
\hline
\end{tabular}

\subsubsection{Risk factors related to breastfeeding}


As for the relationship between breastfeeding posture and LM, the pooled result of three trials $[23,26,27]$ showed that improper breastfeeding posture or laid-back breastfeeding was identified as a significant risk factor for LM (OR $2.47,95 \% \mathrm{Cl}[2.09,2.92], l^{2}=0 \%, 3$ trials, PAR $26.51 \%)$.

\subsubsection{Milking method}

As for the relationship between milking method and LM, two studies were included in this analysis [23, 26], and the pooled result showed that the improper milking method during breastfeeding was identified as an important risk factor for LM (OR 6.79, 95\% $\mathrm{Cl}[3.45$, 13.34], $I^{2}=69 \%, 2$ trials, PAR $\left.59.14 \%\right)$.

\subsubsection{The duration of each breastfeeding}

As for the relationship between the duration of each breastfeeding and LM, six trials $[20,22,24,25,27,28]$ were included in this analysis, and the pooled result showed that the duration of each breastfeeding $\mathbb{0} 0.5 \mathrm{~h}$ was not recognized as the risk factor for LM (OR $0.77,95 \% \mathrm{Cl}[0.48,1.24], l^{2}=83 \%, 6$ trials, PAR not calculable).

\subsubsection{The way baby sucks nipple}

As for the relationship between the way of baby sucking nipple and LM, seven trials [22-24,26-29] were included in this analysis, and the pooled result showed that only baby sucking the nipple was not recognized as the risk factor for $\mathrm{LM}(\mathrm{OR} 0.76,95 \% \mathrm{Cl}[0.19,2.97]$, $I^{2}=98 \%, 7$ trials, PAR not calculable).

\subsubsection{Repeated milk stasis}

As for the relationship between repeated milk stasis during breastfeeding and LM, the result of two studies [24, 25] showed that repeated milk stasis during breastfeeding was identified as a significant risk factor for LM $\left(\mathrm{OR} 6.08,95 \% \mathrm{Cl}[3.75,9.88], I^{2}=36 \%, 2\right.$ trials, PAR $37.42 \%)$.

\subsubsection{Nipple cleaning condition}

As for the relationship between nipple cleaning condition and LM, seven trials were included $[20,22-25,27,28]$ in this analysis, and the pooled result showed that little or no cleaning nipple during breastfeeding was identified as a significant risk factor for LM (OR 2.38, $95 \% \mathrm{Cl}[1.88,3.03], I^{2}=52 \%, 7$ trials, PAR $\left.31.28 \%\right)$.

\subsubsection{Risk factors related to maternity}

\subsubsection{Abnormal nipple or crater nipple}

As for the relationship between abnormal nipple/crater nipple and LM, the pooled result of four studies $[20,21,25,29]$ showed that mother with abnormal nipple or crater nipple experienced a higher risk of LM (OR 2.94, 95\% $\mathrm{Cl}[1.76,4.91], l^{2}=76 \%, 4$ trials, PAR $\left.33.99 \%\right)$.

\subsubsection{Primipara}

As for the relationship between the times of women's delivery and LM, the pooled result of five studies $[20,21,24,26,29]$ showed that primipara experienced a higher risk of LM $(\mathrm{OR} 1.91,95 \% \mathrm{Cl}[1.26,2.90], l=60 \%, 5$ trials, PAR $39.06 \%)$. Similarly, the result from one cross sectional study [30] involving 864 participants reported that mother with primipara experienced a higher risk of LM $(O R 3.46,95 \% \mathrm{Cl}$ $[1.04,11.46])$.

\subsubsection{History of breast trauma}

As for the relationship between history of breast trauma and LM, the pooled result of six trials $[22-25,27,28]$ showed that mother with the history of breast trauma experienced a higher risk of LM (OR 3.57, $95 \% \mathrm{Cl}[2.86,4.46], l^{2}=29 \%, 6$ trials, PAR $\left.13.36 \%\right)$.

\subsubsection{History of breast disease}

As for the relationship between history of breast disease and $L M$, the result of four studies $[23,25,26,29]$ showed that history of breast disease was not identified as a significant risk factor for LM (OR $0.99,95 \% \mathrm{Cl}[0.41,2.42], I^{2}=93 \%, 4$ trials, PAR not calculable). 
As for the relationship between history of diabetes and LM, the result of three studies $[21,24,25]$ showed that history of diabetes was found to be associated with LM (OR 2.26, 95\%CI [1.43, 3.58], $I^{2}=46 \%, 3$ trials, PAR $\left.6.81 \%\right)$.

\subsubsection{Method of delivery}

As for the relationship between the method of delivery and LM, the result of three studies $[20,24,29]$ showed that experience of cesarean section was identified as a significant risk factor for $\operatorname{LM}\left(\mathrm{OR} 1.77,95 \% \mathrm{Cl}[1.32,2.38],{ }^{2}=38 \%, 3\right.$ trials, PAR $28.34 \%$ ).

\subsubsection{Education level}

As for the relationship between the education level and $L M$, the result of four studies $[20,23,25,26]$ showed that mother with the low education level (high school or below) experienced a higher risk of LM (OR 1.85, 95\% Cl [1.04, 3.28], $l^{2}=82 \%, 4$ trials, PAR $23.67 \%$ ). Similar result was found in one cross sectional study [30] involving 864 participants (OR $2.2,95 \% \mathrm{Cl}[1.11,4.35])$.

\subsubsection{Breast massage experience of non-medical personnel}

As for the relationship between breast massage experience of non-medical personnel and LM, the result of two studies [24, 27] showed that mother with breast massage experience of non-medical personnel experienced a higher risk of LM $(\mathrm{OR} 1.90,95 \% \mathrm{Cl}[1.36,2.65]$, $I^{2}=24 \%, 2$ trials, PAR $\left.20.89 \%\right)$.

\subsubsection{Other risk factors related to postpartum period}

\subsubsection{Six months after delivery}

As for the relationship between postpartum period and LM, the result of five studies $[22,23,25,27,28]$ showed that postpartum within 6 months was identified as the risk factor for LM (OR 5.11, 95\% CI [2.66, 9.82], $l^{2}=93 \%, 5$ trials, PAR $\left.65.93 \%\right)$.

\subsubsection{Sleeping posture after delivery}

As for the relationship between sleeping posture after delivery and LM, the result of four studies $[22-24,28]$ showed that mother with the prone sleeping position after delivery experienced a higher risk of LM (OR 2.53, 95\% CI [1.51, 4.22], $I^{2}=84 \%, 4$ trials, PAR $\left.22.72 \%\right)$. Similar result was found in one cross sectional study [31] involving 68 participants (OR 2.26, 95\% $\mathrm{Cl}[1.23,4.11])$.

\subsubsection{Rest time of postpartum women}

As for the relationship between rest time of postpartum women and LM, the result of two studies $[23,26]$ showed that postpartum rest time less than 3 months was identified as a risk factor for LM (OR 4.71,95\% Cl [3.92, 5.65], $l^{2}=0 \%, 2$ trials, PAR 56.95\%) .

\subsubsection{Postpartum bad mood}

As for the relationship between bad mood after delivery and LM, the result of seven studies $[20,22-24,26-28]$ showed that mother with the bad mood after delivery was found to seem not significantly associated with LM (OR $1.28,95 \% \mathrm{Cl}[0.92,1.79], l^{2}=77 \%, \mathrm{n}=7$ trials, PAR not calculable).

\subsection{Sensitivity analysis}

Sensitivity analysis was performed by eliminating each study one by one, at a time the summary $\mathrm{P}$ values and ORs of the remaining studies were recalculated. The results of each breastfeeding duration $>0.5 \mathrm{~h}$, the way baby sucking nipple and postpartum bad mood partially deviated from the $95 \%$ confidence interval estimated by meta-analysis, indicating that the robustness of the current available data for these factors was poor. The pooled results of these risk factors may be influenced by high risk bias trials (Fig.2, Fig.3 and Fig. 4). The robustness of meta-analysis for other risk factors is acceptable.

\subsection{The analysis of Nfs and PAR}

$\mathrm{Nfs}$ estimates of the risk factors were created with the formula obtained from the data analysis section. The results of $\mathrm{Nfs}_{0.05}$ showed that if another 16 studies were negative, the history of diabetes would be not related to LM. Similarly, the results of $\mathrm{Nfs}_{0.05}$ showed that 
if another 14 studies were negative, history of breast disease would not be related to LM. Nfs estimates for other risk factors illustrated that there were good robustness of the pooled results and publication bias had no significant influence on current results.

The estimates of PAR indicated that only about $6.81 \%$ of LM in this population can be attributed to history of diabetes, indicating a relatively low chance of exposure in this population. However all of other risk factors (OR囚1) had a great impact on the incidence of LM in Chinese women, and had a high chance of exposure in this population. The results of Nfs and PAR\% were showed in Table 3.

\subsection{Publication bias}

Additionally, Egger's linear regression analysis was based on trial data that reported risk factors for little or no nipple cleaning. The result of Egger's test (Std. Err $=1.589494, t=0.52, P>|t|=0.627)$ demonstrated that the included studies may have potential publication bias (Fig. 5).

\section{Discussion}

LM has serious physiological and psychological effects on breastfeeding mothers. In order to obtain more meaningful data on the significant risk factors of $L M$, we conducted a comprehensive meta-analysis based on published studies. Twelve studies were included in this review, involving a total of 6539 participants. The results demonstrated that the following risk factors were identified as significant risk factors for LM. (1) The risk factors that were categorized as breastfeeding related included improper breastfeeding posture, improper milking method, repeated milk stasis during breastfeeding and little or no cleaning nipple. (2) The risk factors that were categorized as maternal related included abnormal nipple or crater nipple, primipara, history of breast trauma, experience of cesarean section, low education level and breast massage experience of non-medical personnel. (3) Risk factors associated with postpartum period included postpartum within 6 months, the prone sleeping position after delivery and postpartum rest time less than 3 months. These findings were consistent with the results of the previous studies.

In order to estimate the potential impact of risk factors on LM at the population level and better guide clinical practice, we calculated PAR percent for the risk factors significantly associated with LM. In particular, mothers with history of diabetes were reported to significantly associated with $L M$, However, since the exposure rate $(P A R=6.81 \%)$ was relatively low and only three studies were included in the analysis, further studies are needed to confirm this finding. Other high risk factors (OR囚1) had high to medium chance of exposure in the general population.

In this study, the pooled result found that the duration of lactation $>0.5 \mathrm{~h}$ seemed to be unrelated to $\mathrm{LM}$, which was inconsistent with previous studies $[20,25]$. In addition, the sensitivity analysis of this result indicated that the robustness of the current available data was relatively poor. Therefore, the further studies on this topic are recommended to confirm whether it is a risk factor related to LM. Some researchers have found that a history of mastitis has been identified as a plausible risk factor of LM [29, 32]. However, this study did not find a link between the history of breast disease and LM, which may be related to the fact that the disparities in reported effect sizes among included studies may be attributed to the differences in the underlying population, definition of disease and case definition adopted. Thus there is still insufficient evidence allowing us to draw a conclusion on this factor.

Previous research have found that preterm infants have an immature sucking behavior, which may have influence on the capacity of exclusively breastfed for a period of weeks or months [33]. Similar to previous studies [24, 27, 29], the way baby sucking nipple (only sucking the nipple) was found to be another risk factor associated with LM. In addition, the Department of Maternal and Child, China's Ministry of Health has issued a breastfeeding manual, which encourages infants to suck nipples and areola during breast-feeding [22]. However, the results of this review showed that there was no significant relationship between sucking patterns and LM, so it was not possible to determine the effect of nipple sucking, and further studies were recommended.

Studies conducted by some scholars $[10,14,16,17]$ have found that maternal bad mood is associated with LM. Similar to previous studies [34], maternal stress was identified as a factor associated with LM in another study. In addition, it is reported that negative emotions can significantly reduce the body's "SIgA" level, which will lead to a decline in the body's resistance to bacteria [35]. Therefore, maternal bad mood may be a risk factor for LM [22]. However, this review did not find a significant relationship between maternal bad mood and LM, which may be related to the inconsistent severity and definition of adverse emotions in different studies. Accordingly, the multidisciplinary team involved in maternal and infant breastfeeding management should be aware of the possibility of LM in mothers with bad mood, especially those with a history of mental health problems [36]. 
This review, to the best of our knowledge, is the first meta-analysis to explore the high risk factors for LM by synthesizing the available information. The findings might provide evidence-based information for the high risk factors of LM. Most importantly, this review provides a reference for the prevention of LM and further study on the pathogenic factors of LM.

There is no denying that this study has some limitations. Firstly, the disparities in heterogeneity among studies may have affected the effectiveness of statistical analysis, due to potential confounding factors such as sample size, design differences, underlying population characteristics, etc. Secondly, the potential publication bias found in this review may be attributed to the limited overlap of high risk factors between studies. As a result, further studies need to be conducted to accumulate the evidence results on each risk factor. Finally, the effect estimates could not be calculated for all risk factors, because more than two studies related to the same defined risk factor for LM were pooled in the meta-analysis. However, our findings made an important contribution to determining the well-accepted risk factors related to LM by integrating studies of LM risk factors, and specified the aspects that need to be investigated in the future.

\section{Conclusions}

The significant risk factors for LM were improper breastfeeding posture, improper milking method, repeated milk stasis, little or no cleaning of nipples, abnormal nipple or crater nipple, primipara, history of breast trauma, experience of cesarean section, low educational level, breast massage experience of non-medical personnel, postpartum within 6 months, the prone sleeping position after delivery and postpartum rest time less than 3 months. These findings have some reference value for the prevention and treatment of LM. In particular, by controlling some of modifiable factors such as breastfeeding posture, milking method, milk stasis situation and nipple cleaning condition, the incidence of LM may be reduced. Providing guidance to mothers on how to deal with bad mood seems also important to prevent LM.

\section{Abbreviations}

Cl: confidence intervals; RCT: randomized controlled trial; OR: odds ratio; REM: random effect model; FIX: fixed effect model; Nfs: failsafe number; T: Case group, C: Control group, NR: Not reported; NA: not available; LM: Lactation mastitis; WHO: the World Health Organization; PAR: the population attributable risks percent; NOS: the criteria of Newcastle-Ottawa Scale; ARHQ: The Agency for Healthcare Research and Quality.

\section{Declarations}

\section{Acknowledgments}

We would like to thank all authors of the primary studies included in this review.

\section{Author's contribution}

Bao-Yong Lai, Xiao-Hua Pei designed the review. Bao-Yong Lai, Li-Yan Jia participated in searching, selecting trials, and extracting data. Li-Yan Jia participated in assessing the qualities of included studies. Bao-Yong Lai, Ai-Jing Chu contributed to performing data analyses and first draft of manuscript. Bo-Wen Yu, Shi-Bing Liang, Jian-Ping Liu, Xiao-Hua Pei were all involved in critically revising the manuscript. All authors have read and approved the final manuscript.

\section{Funding}

This work is supported by the fund from the Project and Study on clinical evaluation of traditional Chinese medicine in reducing the use of antibiotics during the treatment of acute suppurative mastitis (No. Shou Fa 2018-7031).

\section{Availability of data and materials}

The datasets used and/or analyzed during the current study are available from the corresponding author on reasonable request.

\section{Ethics approval and consent to participate}

Not applicable. 


\section{Consent for publication}

Not applicable.

\section{Competing interests}

All authors declare that they have no competing interest.

\section{References}

1. World Health Organization. Mastitis: Cause and Management. Publication Number WHO/FCH/CAH/00.13.WorldHealth Organization, Geneva, 2000.

2. Amir LH. Academy of Breastfeeding Medicine Protocol Committee. ABM clinical protocol \#4: Mastitis, revised March 2014. Breastfeed Med. 2014; 9(5):239-243.

3. Kinlay JR, O'Connell DL, Kinlay S. Incidence of mastitis in breastfeeding women during the six months after delivery: a prospective cohort study. Med J Aust. 1998; 169(6):310-312.

4. Zarshenas M, Zhao Y, Poorarian S, Binns CW, Scott JA. Incidence and Risk Factors of Mastitis in Shiraz, Iran: Results of a Cohort Study. Breastfeed Med. 2017; 12:290-296.

5. Dixon JM, Khan LR. Treatment of breast infection. BMJ. 2011; 342:d396.

6. Bolman M, Saju L, Oganesyan K, Kondrashova T, Witt AM. Recapturing the art of therapeutic breast massage during breastfeeding. J Hum Lact. 2013; 29(3):328-331.

7. Bond DM, Morris JM, Nassar N. Study protocol: evaluation of the probiotic Lactobacillus Fermentum CECT5716 for the prevention of mastitis in breastfeeding women: a randomized controlled trial. BMC Pregnancy Childbirth. 2017; 17(1):148.

8. Victora CG, Bahl R, Barros AJD, França GVA, Horton S, Krasevec J, et al. Breastfeeding in the 21st century: epidemiology, mechanisms, and lifelong effect. The Lancet. 2016; 387(10017):475-490.

9. Bolton KA, Kremer P, Hesketh KD, Laws R, Kuswara K, Campbell KJ. Differences in infant feeding practices between Chinese-born and Australian-born mothers living in Australia: a cross-sectional study. BMC Pediatr. 2018; 18(1):209.

10. Wang L, Li QJ, Miao XF, Chen H, Zhao Y, Liu LY, et al. Correlation between types of feeding and growth and nutrition status of 42day infants. Chinese Journal of Reproductive Health. 2020; 31(01):24-27.

11. Qiu C. The status of weight and length growth and feeding patterns of 323 infants aged 1-2 months in Beijing. Chinese Journal of Women and Children Health.2020; 11(02):14-17.

12. Leung SS. Breast pain in lactating mothers. Hong Kong Med J. 2016; 22(4):341-346.

13. Amir LH, Forster DA, Lumley J, McLachlan H. A descriptive study of mastitis in Australian breastfeeding women: incidence and determinants. BMC Public Health. 2007; 7(1):62.

14. Cooklin, A.R., Amir, L.H, Nguyen, C.D, CASTLE Study Team et al. (2018) Physical health, breastfeeding problems and maternal mood in the early postpartum: a prospective cohort study. Arch. Womens Ment. Health 21, 365-374.

15. Olga Pustotina. Management of mastitis and breast engorgement in breastfeeding women [J]. J Matern Fetal Neonatal Med, 2016, 29(19):3121-3125.

16. Stang A. Critical evaluation of the Newcastle-Ottawa scale for the assessment of the quality of nonrandomized studies in metaanalyses. Eur J Epidemiol. 2010; 25(9):603-605.

17. Zeng X, Zhang Y, Kwong JS, et al. The methodological quality assessment tools for preclinical and clinical studies, systematic review and meta-analysis, and clinical practice guideline: a systematic review. J Evid Based Med. 2015; 8(1):2-10.

18. Doidge JC, Segal L, Gospodarevskaya E. Attributable risk analysis reveals potential healthcare savings from increased consumption of dairy products. J Nutr. 2012; 142(9):1772-1780.

19. Fragkos KC, Tsagris M, Frangos CC. Publication Bias in Meta-Analysis: Confidence Intervals for Rosenthal's Fail-Safe Number. Int Sch Res Notices. 2014; 2014:825383.

20. Tang L, Lee AH, Qiu L, Binns CW. Mastitis in Chinese breastfeeding mothers: a prospective cohort study. Breastfeed Med. 2014; 9(1):35-38.

21. Yu Z, Sun S, Zhang Y. High-Risk Factors for Supprative Mastitis in Lactating Women. Med Sci Monit. 2018; 24:4192-4197.

Page $13 / 19$ 
22. He XP, Ma XJ, Zhao YZ, Gao HF, He Y. Correlation analysis between the patients' behaviors and acute lactational mastitis. Chinese Journal of Breast Disease (Electronic Edition). 2013; 7(01):39-42.

23. Pu YN. Study on the incidence and influencing factors of acute mastitis during lactation in a hospital from 2011 to 2015 . Chinese Journal of Hospital Statistics.2017; 24(04):288-290.

24. Li JX, Xiang GY, Lin JP. Analysis on the risk factors related to lactation mastitis. Guangdong Medical Journal. 2019; 40(S1):281283.

25. Wang HM, Huang XX, Chen ZW, Wu JB, ZHai S, Peng Y. Analysis on the risk factors related to developing lactation mastitis. Journal of Fujian Medical University. 2016; 50(06):423-427.

26. Cheng MH, Li SY, Duan Y. Analysis on related factors of lactation mastitis in women in Bao'an region Shenzhen City and nursing countermeasures. Chinese General Practice Nursing. 2014; 12(05):385-386.

27. ZHai HL. Analysis on the risk factors related to acute lactational mastitis and treatment strategies. Journal of Community Medicine. 2017; 15(21):22-24.

28. Gao X, Lv G. Analysis on related factors of acute lactational mastitis and treatment strategies. Guangdong Medical Journal. 2015; 36(20):3210-3212.

29. Chen XG. Risk factors of staphylococcus aureus infection in acute mastitis. Shenzhen Journal of Integrated Traditional Chinese and Western Medicine. 2016; 26(12):101-103.

30. Xia HL, Yang LC. Risk factors for puerperal mastitis. Anhui Medical and Pharmaceutical Journal. 2011; 15(12):1522-1524.

31. Wang CL. Analysis on the risk factors and treatment strategies related to lactation mastitis. Home Medicine. 2018; (10):335.

32. Kinlay JR, O' Connell DL, Kinlay S. Risk factors for mastitis in breastfeeding women: Results of a prospective cohort study. Aust N Z J Public Health. 2001; 25:115-120.

33. Flacking R, Dykes F. Perceptions and experiences of using a nipple shield among parents and staff-an ethnographic study in neonatal units. BMC Pregnancy Childbirth. 2017; 17(1):1.

34. Fetherston C. Risk factors for lactation mastitis. J Hum Lact. 1998; 14:101-109.

35. Zhang W, Li F, Qin S, Luo J. The integrative effects of cognitive reappraisal on negative affect: associated changes in secretory immunoglobulin A, unpleasantness and ERP activity. PLoS One. 2012; 7(2):e30761.

36. Sun K, Chen M, Yin Y, Wu L, Gao L. Why Chinese mothers stop breastfeeding: Mothers' self-reported reasons for stopping during the first six months. J Child Health Care. 2017; 21(3):353-363.

\section{Figures}




\begin{tabular}{|c|c|c|c|c|c|c|}
\hline $\begin{array}{c}\text { VIP } \\
\mathrm{n}=38\end{array}$ & $\begin{array}{c}\text { Wanfang } \\
\mathrm{n}=61\end{array}$ & $\begin{array}{l}\mathrm{CNKI} \\
\mathrm{n}=63\end{array}$ & \multicolumn{2}{|c|}{$\begin{array}{c}\text { SINOMED } \\
\mathrm{n}=35\end{array}$} & $\begin{array}{c}\text { Web of science } \\
n=4\end{array}$ & $\begin{array}{c}\text { PubMed } \\
n=14\end{array}$ \\
\hline \multicolumn{7}{|c|}{$\downarrow$} \\
\hline \multicolumn{4}{|c|}{ Recorders after 53 duplicates removed $(n=162)$} & \multirow{3}{*}{\multicolumn{3}{|c|}{$\begin{array}{l}\text { Recorders excluded ( } \mathrm{n}=136 \text { articles): } \\
\text { Due to unrelated research, reviews, } \\
\text { animal experiments, duplicate studies } \\
\text { and other non-conforming studies. }\end{array}$}} \\
\hline & & Title & abstract & & & \\
\hline & & $\downarrow$ & & & & \\
\hline \multicolumn{4}{|c|}{ Recorders after the title and abstract $(\mathrm{n}=26)$} & & & \\
\hline & & Full & xt reading & \multirow{3}{*}{\multicolumn{3}{|c|}{$\begin{array}{l}\text { Recorders excluded ( } \mathrm{n}=14 \text { articles): } \\
\text { Due to studies involving non-lactating } \\
\text { mastitis, improper study design type, } \\
\text { duplicate recorders. }\end{array}$}} \\
\hline & & $\downarrow$ & & & & \\
\hline \multicolumn{4}{|c|}{ Trials included for analysis $(\mathrm{n}=12)$} & & & \\
\hline
\end{tabular}

Figure 1

study selection flow diagram 


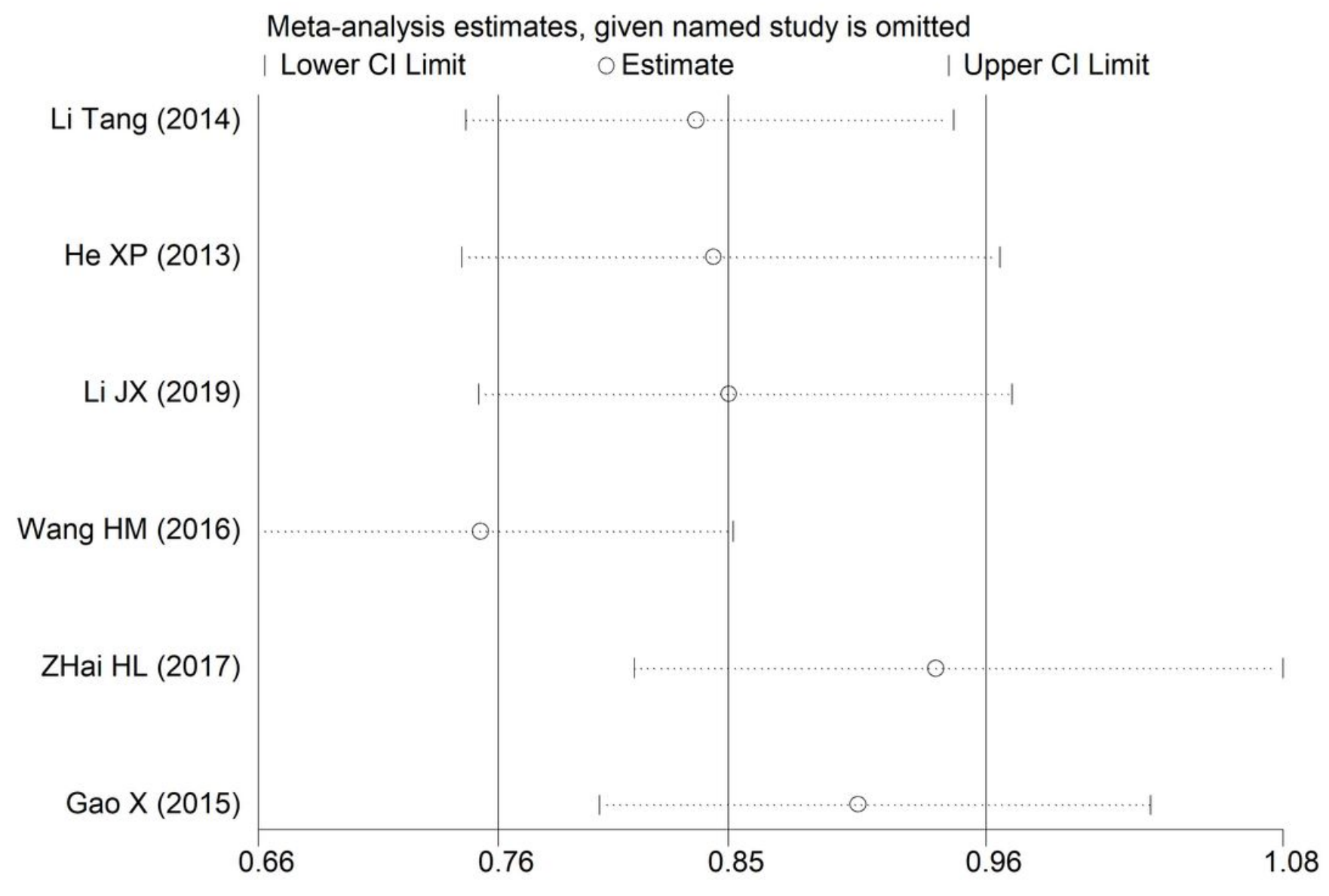

Figure 2

sensitivity analysis for the duration of each breastfeeding $₫ 0.5 \mathrm{~h}$ 
Meta-analysis estimates, given named study is omitted

| Lower Cl Limit | Estimate Upper Cl Limit

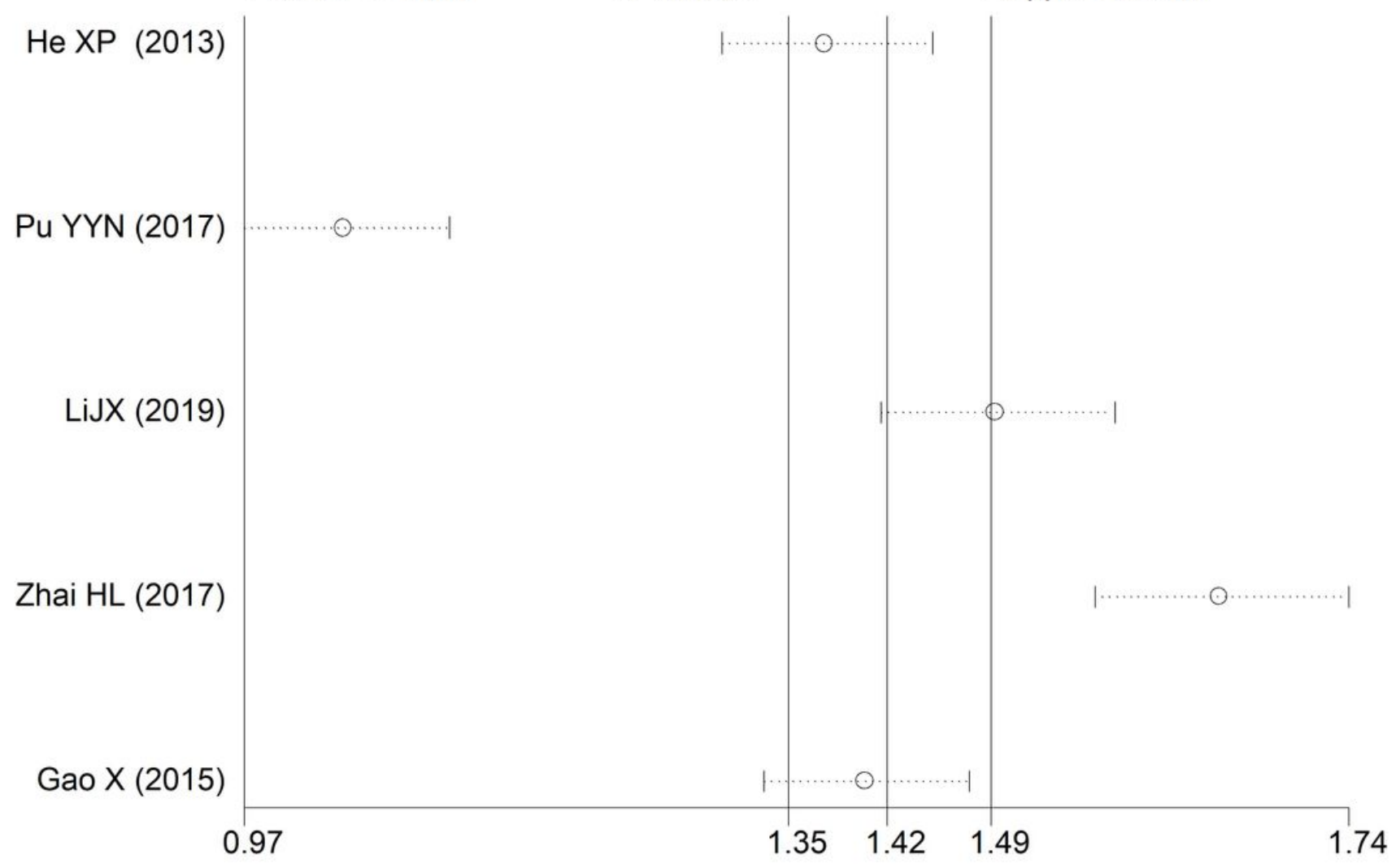

Figure 3

sensitivity analysis for the way of baby sucking nipple 
Meta-analysis estimates, given named study is omitted

| Lower Cl Limit

I Upper Cl Limit

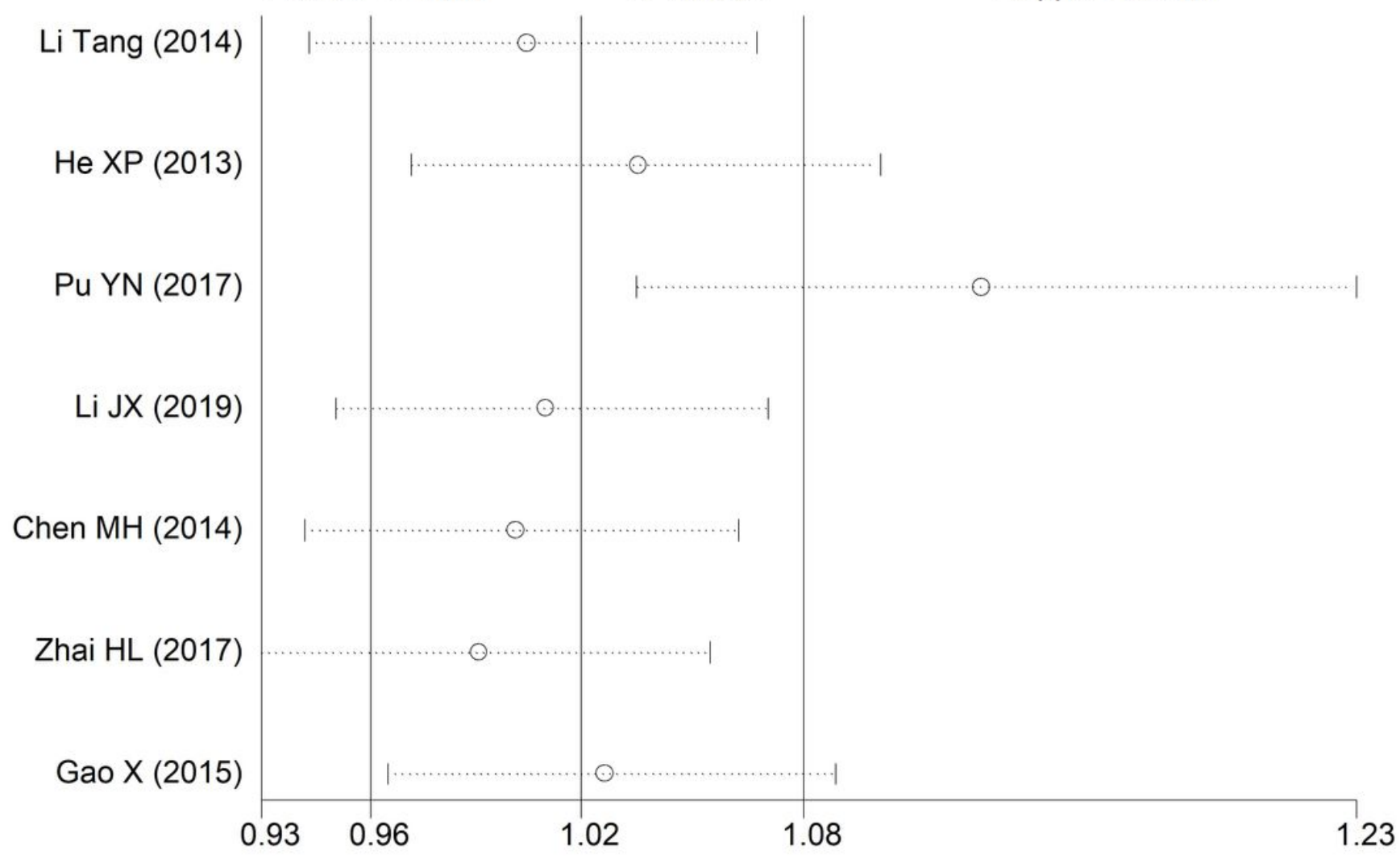

Figure 4

sensitivity analysis for postpartum bad mood 


\section{Egger's publication bias plot}

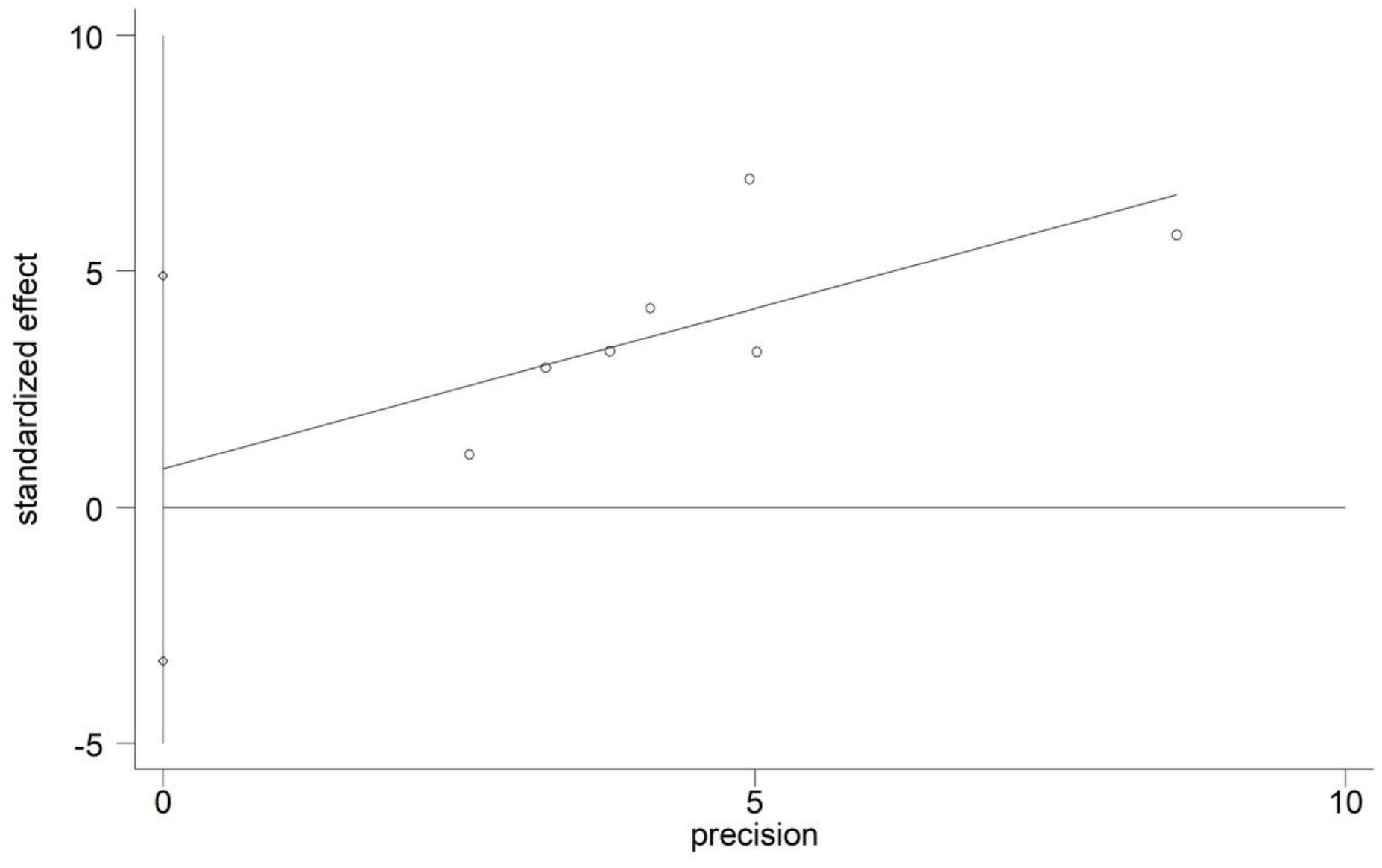

Figure 5

Egger's publication bias plot 